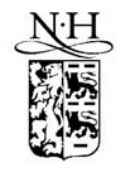

ELSEVIER

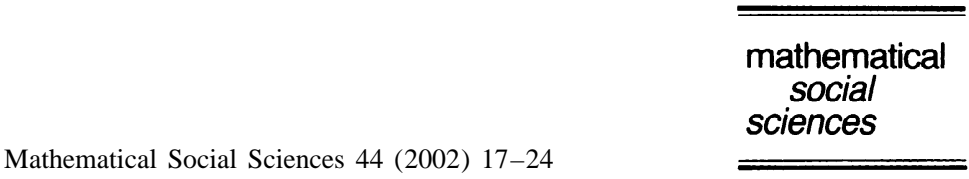

www.elsevier.com/locate/econbase

\title{
A remark on bargaining and non-expected utility
}

\author{
Oscar Volij* \\ Department of Economics, Iowa State University, Ames, IA 50011, USA
}

Received 1 October 2001; received in revised form 1 January 2002; accepted 1 March 2002

\begin{abstract}
We show that a bargaining game of alternating offers with exogenous risk of breakdown and played by dynamically consistent non-expected utility maximizers is formally equivalent to Rubinstein's [Econometrica 50 (1982) 97] game with time preference. Within this game, the behavior of dynamically consistent players is indistinguishable from the behavior of expected utility maximizers.

(C) 2002 Elsevier Science B.V. All rights reserved.

Keywords: Bargaining; Compound lotteries; Dynamic consistency

JEL classification: C70; C72; C78
\end{abstract}

\section{Introduction}

Nash's (1950) axiomatic model of two-person bargaining assumes that the players have expected utility preferences. Several other papers have shown, however, that the axiomatic bargaining theory can be extended to allow for some non-expected utility preferences. Within the strategic approach, the early models do not involve risk preferences but time preferences. Binmore et al. (1986) show, however, that a bargaining game of alternating offers and exogenous risk of breakdown is formally equivalent to the bargaining model with time preferences, at least when players preferences satisfy the expected utility axioms.

Extending a noncooperative bargaining model to non-expected utility preferences

*Corresponding author. Tel.: + 1-515-294-7909; fax: + 1-515-294-0221.

E-mail address: oscar@volij.co.il (O. Volij).

${ }^{1}$ Following the seminal paper by Rubinstein et al. (1992), one can find Grant and Kajii (1995), Hanany and Safra (2000), Safra and Zilcha (1993) and Volij and Winter (2002) among many others. 
requires some modeling choices. Since a pair of strategies induces a compound lottery over terminal histories, one needs to decide what kind of preferences over compound lotteries to allow. Segal (1990) showed that if one is to leave the expected utility model, then one needs to drop either the compound independence axiom or the reduction of compound lotteries axiom. Both axioms are appealing and none of them appears to be a clear choice at first sight. Both approaches have been used in the past, in the context of strategic form games. Crawford (1990) introduces the notion of equilibrium in beliefs, which coincides with the Nash equilibrium concept under the expected utility hypothesis. He adopts the reduction of compound lotteries axiom and shows conditions under which an equilibrium in beliefs does exist in a game. Dekel et al. (1991) adopt the compound independence axiom and show that under certain conditions a Nash equilibrium exists in every strategic form game. Volij (1996) extends Aumann and Brandenburger's (1995) results on epistemic conditions for Nash equilibrium both for the case when preferences satisfy compound independence and the reduction axiom.

In the context of extensive form games, compound independence seems to be more appealing than the reduction axiom because it guarantees that players' behavior is dynamically consistent, a property that is vacuous in static games. The dynamic inconsistency of a player's behavior has serious consequences for existence of subgame perfect equilibrium: in some finite games subgame perfect equilibria may fail to exist. This is due to the fact that when players behave in a dynamically inconsistent way, the one deviation property does not hold in general. Having said that, the two-person bargaining model where players' preferences satisfy the reduction of compound lotteries axiom does have a subgame perfect equilibrium, which was characterized by Burgos et al. (2002).

In this paper we extend Binmore et al. (1986) result to preferences that satisfy the compound independence axiom. In fact we show that under this assumption, and within the noncooperative bargaining model, the resulting preferences are indistinguishable from expected utility ones. Therefore all the results known about the bargaining model of alternating offers are robust to changes in the risk preferences, as long as these preferences satisfy the compound independence axiom.

The paper is organized as follows. We first briefly describe Rubinstein's (1982) game of alternating offers with a constant risk of breakdown. We complete the description of the game by concentrating on a small class of compound lotteries and restricting preferences over that class to satisfy several properties, compound independence being the crucial one. We make a short detour and show that without compound independence the one deviation property may fail, and thus subgame perfect equilibrium may fail to exist even in finite games. We then go back to the analysis of the game and prove that it is equivalent to the standard Rubinstein game with time preferences and no risk of breakdown. We end with a remark on the generality of the bargaining model with non-expected utility preferences.

\section{Rubinstein's game of alternating offers with a constant risk of breakdown}

There are two players and a perfectly divisible object of size 1 . The possible divisions of the object are thus given by 


$$
X=\left\{\left(x_{1}, x_{2}\right) \in K_{+}^{2}: x_{1}+x_{2}=1\right\}
$$

Consider the following game form. Players alternate in making proposals from the set $X$. After a proposal is made by one player, the other player responds by either accepting or rejecting it. If the offer is accepted, the game ends and the accepted proposal is implemented. If the proposal is rejected, then with a fixed probability $1-\delta$ the game ends and the division is $(0,0)$, and with probability $\delta$ the game continues with a new proposal made by the previous responder. A strategy for player $i=1,2$ is a specification of a feasible action for every node at which player $i$ is called to play. That is, when $i$ is supposed to propose, the strategy specifies an element of $X$ and when $i$ is supposed to respond to a proposal $x \in X$, a strategy specifies whether or not to accept $x$. Strategies are not restricted to be stationary. A strategy profile is a pair $\sigma=\left(\sigma_{1}, \sigma_{2}\right)$ of strategies, one for each player. A pair of strategies uniquely determines a compound lottery. Therefore, in order to define the players' preferences over lotteries on terminal histories and thus complete the definition of the game, it is enough to define their preferences over compound lotteries. Further, since the set of strategy profiles induces only a small set of compound lotteries over $X$, it is enough to define the players' preferences only on that small space of lotteries.

\subsection{Compound lotteries}

An elementary lottery over a set $Z$ is defined to be a lottery that awards an element $z \in Z$ with probability $p$, and $(0,0)$ with the complementary probability. We denote such a lottery by $\langle z, p\rangle$. As a consequence, the set of elementary lotteries over $X$, or one-stage lotteries, is given by

$$
\mathbf{L}^{1}=\{\langle x, p\rangle: x \in X, p \in[0,1]\} .
$$

For each division $x \in X$ we identify $x$ with $\langle x, 1\rangle \in \mathbf{L}^{1}$. We now define compound lotteries over $X$, recursively, as follows: for all $n=2,3, \ldots$, an $n$-stage lottery is an elementary lottery over the set of $(n-1)$-stage lotteries. Formally,

$$
\mathbf{L}^{n}=\left\{\left\langle\ell^{n-1}, p\right\rangle: \ell^{n-1} \in \mathbf{L}^{n-1}, p \in[0,1]\right\} \quad n=2,3, \ldots
$$

The set of finite compound lotteries is then

$$
\mathbf{L}=\bigcup_{n=1}^{\infty} \mathbf{L}^{n}
$$

We shall represent the $n$-stage lottery $\ell=\left\langle\ldots\left\langle\left\langle x, p_{1}\right\rangle, p_{2}\right\rangle, \ldots, p_{n}\right\rangle$ more succinctly by $\left\langle x,\left(p_{k}\right)_{k=1}^{n}\right\rangle$. We can, thus, think of any lottery $\ell \in \mathbf{L}^{n}$ as a point in $\mathbb{R}^{n+1}$ with its associated Euclidean topology.

There are strategy pairs, $\sigma$, that induce finite compound lotteries. Note that these lotteries are of the form $\ell=\langle\ldots\langle\langle x, \delta\rangle, \delta\rangle, \ldots, \delta\rangle=\left\langle x,(\delta)_{k=1}^{t}\right\rangle \in \mathbf{L}$. There are strategy pairs, on the other hand, that do not induce a finite compound lottery but an infinite one. For example a pair of strategies that induces eternal rejection of the proposed divisions, induces an infinite compound lottery. We shall denote this infinite compound lottery by $D$. Note that $D$ is the only infinite lottery that can be induced in this game. 
Player $i=1,2$ is supposed to have a preference relation $\succeq_{i}$ over elements of $\mathbf{L} \cup\{D\}$, that satisfy the following properties:

A1. No agreement is worse than disagreement. For all $\ell \in \mathbf{L}, \ell \succeq_{i} D$.

A2. Compound independence. For all $\ell, \ell^{\prime} \in \mathbf{L}$ and for all $p \in[0,1], \ell \succeq_{i} \ell^{\prime}$ if and only if $\langle\ell, p\rangle \succeq_{i}\left\langle\ell^{\prime}, p\right\rangle$.

A3. Monotonicity. For all $x \in X$ and $p \in[0,1], x \succeq\langle x, p\rangle$. For all $x, y \in X$ and $i=1,2$, if $x_{i}>y_{i}$ then $x>{ }_{i} y$.

A4. Continuity. If for all $n \in \mathbb{N}$ and for some fixed $k$ and $s, \ell_{n} \in \mathbf{L}^{k}$ and $\ell_{n}^{\prime} \in \mathbf{L}^{s}$ are such that $\ell_{n} \succeq_{i} \ell_{n}^{\prime}$, and both $\left\{\ell_{n}\right\}$ converges to $\ell \in \mathbf{L}^{k}$ and $\left\{\ell_{n}^{\prime}\right\}$ converges to $\ell^{\prime} \in \mathbf{L}^{s}$, then $\ell \succeq_{i} \ell^{\prime}$.

The first requirement is a standard requirement in bargaining theory. Monotonicity is monotonicity with respect to first order stochastic dominance. Continuity is a standard technical requirement. The most important property is compound independence, which imposes dynamic consistency on the players' behavior. Compound independence is convincingly advocated for in Segal (1990). Dekel et al. (1991) use this approach to show existence of Nash equilibrium in normal form games where players are not expected utility maximizers. Further, it can be shown that provided players satisfy compound independence, all finite extensive form games with chance moves satisfy the one-deviation property and thus Kuhn's theorem extends to this larger domain (see Osborne and Rubinstein (1994, pp. 98-99) for the statements and proofs of the one-deviation property and Kuhn's theorem).

It should be noted that compound independence does not impose any restriction on risk preferences over simple lotteries. In particular, consider the preferences defined as follows. Let $v$ be an arbitrary continuous and increasing real function that maps $[0$, $1] \times[0,1]$ into $[0,1]$. Say that compound lottery $\left\langle x,\left(p_{i}\right)_{i=1}^{n}\right\rangle$ is at least as good as compound lottery $\left\langle y,\left(q_{j}\right)_{j=1}^{m}\right\rangle$ if and only if $v\left(\ldots v\left(v\left(x_{1}, p_{1}\right), p_{2}\right), \ldots, p_{n}\right) \geq$ $v\left(\ldots v\left(v\left(y_{1}, q_{1}\right), q_{2}\right), \ldots, q_{m}\right)$. It can be checked that these preferences satisfy compound independence, monotonicity and continuity.

The following claim states that compound independence is a necessary condition for subgame perfect equilibrium to be a non-empty solution concept for finite extensiveform games.

Claim 1. If preferences do not satisfy compound independence one can build finite extensive form games with chance moves that have no subgame perfect equilibrium.

Proof. The reason for the nonexistence of a subgame perfect equilibrium is that when preferences do not satisfy compound independence, even finite games need not have the one-deviation property. To see this, assume that $\succeq$ are continuous preferences that do not satisfy compound independence. Then there are outcomes $y, z \in X$ and probabilities $p, q \in(0,1)$ such that either

- $y\rangle\langle z, p\rangle$ but $\langle\langle z, p\rangle, q\rangle\rangle\langle y, q\rangle$ or

- $\langle z, p\rangle>y$ but $\langle y, q\rangle>\langle\langle z, p\rangle, q\rangle$. 


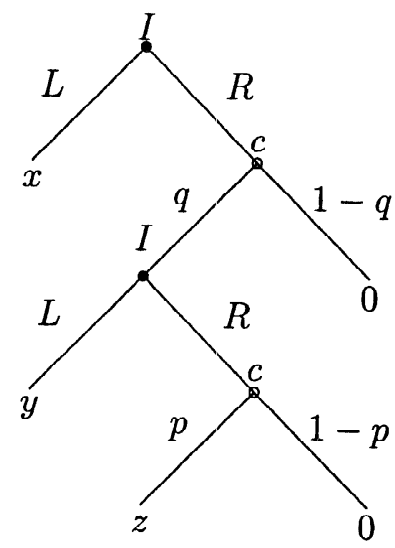

Fig. 1. An extensive game form.

In the first case, let $x \in X$ be an outcome such that $\langle\langle z, p\rangle, q\rangle>x\rangle\langle y, q\rangle$ and consider the game induced by the one-person game form of perfect information and chance moves depicted in Fig. 1.

This game has two subgames: the game itself and the proper subgame that begins at the second decision node. Since a subgame is a game, preferences in the proper subgame are defined accordingly: each strategy in the proper subgame induces a compound lottery and the player's preferences over strategies are those induced by his preferences over compound lotteries. The backward induction strategy is defined inductively: it dictates at the root of each subgame the action that induces the best compound lottery given that in the subsequent nodes play follows the backward induction strategy.

It is easy to see that the backward induction strategy is for the player to choose $L$ at both nodes (because $y\rangle\langle z, p\rangle$ and $x\rangle\langle y, q\rangle$ ). However, the strategy that dictates to choose $R$ at both nodes is a profitable deviation (because $\langle\langle z, p\rangle, q\rangle>x$ ). This means that this game does not have a subgame perfect equilibrium. In the second case, let $x \in X$ be an outcome such that $\langle y, q\rangle>x\rangle\langle\langle z, p\rangle, q\rangle$ and consider the game induced by the same one-person game form of perfect information and chance moves depicted in Fig. 1. It is easy to see that the backward induction strategy is for the player to choose $L$ at the first node and $R$ at the second node (because $\langle z, p\rangle>y$ and $x>\langle\langle z, p\rangle, q\rangle)$. However, the strategy that dictates to choose right at the first node and left at the second node is a profitable deviation (because $\langle y, q\rangle\rangle\langle\langle z, p\rangle, q\rangle$ ). This means that this game does not have a subgame perfect equilibrium.

The example in the above proof is similar to the results that appear in Green (1987) and Wakker (1988), among others. All these papers exploit the dynamic inconsistency of preferences that do not satisfy the compound independence axiom. Also, Claim 1 is related to the discussions that appear in LaValle and Wapman (1986) and Sarin and Wakker (1994) among others. It is shown there that the appropriateness of the application of the folding back procedure to all the equivalent tree-representations of acts, implies the independence axiom. In our case, an individual that satisfies compound 
independence may well not satisfy the independence axiom. But this individual, when evaluating an act, does not apply the folding back technique to all its tree representations but only to one. Still, Claim 1 shows that compound independence is a necessary property to guarantee that the folding back technique yields a subgame perfect equilibrium in all finite extensive form games with chance moves.

It should be noted that Claim 1 does not imply that compound independence is a necessary condition for a non trivial extensive game with chance moves to have a subgame perfect equilibrium. In fact, Burgos et al. (2002) characterize the stationary subgame perfect equilibrium of an alternating offers game where preferences do not necessarily satisfy the compound independence axiom and thus players may behave in a dynamically inconsistent way.

\subsection{Analysis of $\Gamma(\delta)$}

Let $\delta \in(0,1)$ and denote the bargaining game of alternating offers where the constant risk of breakdown is $\delta$, and players' preferences satisfy assumptions $\mathrm{A} 1-\mathrm{A} 4$, by $\Gamma(\delta)$.

Proposition 1. Assume there is a unique pair of agreements $x^{*}$ and $y^{*}$ in $X$ such that

$$
\left\langle x^{*}, \delta\right\rangle \sim{ }_{1} y^{*} \quad \text { and } \quad\left\langle y^{*}, \delta\right\rangle \sim{ }_{2} x^{*} .
$$

Then $\Gamma(\delta)$ has a unique subgame perfect equilibrium which is the following: Player 1 always proposes $x^{*}$ and accepts proposal $y \in X$ if and only $y \succeq_{1} y^{*}$. Player 2 always proposes $y^{*}$ and accepts proposal $x \in X$ if and only $x \succeq_{2} x^{*}$.

Proof. The proof follows from the observation that, given the properties of the players' preferences, especially compound independence, the game $\Gamma(\delta)$ is equivalent to the game of alternating offers analyzed by Rubinstein (1982). To see this, note that each compound lottery $\ell \in \mathbf{L}^{t} \subseteq \mathbf{L}$ that can arise in $\Gamma(\delta)$ can be uniquely associated to a pair $(x, t)$ where $x \in X$ and $t$ is the number of stages in the compound lottery. And vice versa, each pair $(x, t) \in X \times T$ can be uniquely associated to the compound lottery $\left\langle x,(\delta)_{k=1}^{t}\right\rangle$ in $\mathbf{L}$. Therefore, any preference relation $\succeq$ over $\mathbf{L} \cup\{D\}$ induces a preference relation, $\succeq^{\prime}$, over $(X \times T) \cup\{D\}$, where $T=\{0,1, \ldots\}$ represents different time periods, in the following manner:

$$
\begin{aligned}
& (x, t) \succeq^{\prime}(y, s) \quad \Leftrightarrow \quad\left\langle x,(\delta)_{k=1}^{t}\right\rangle \succeq\left\langle y,(\delta)_{k=1}^{s}\right\rangle \\
& (x, t) \succeq^{\prime} D \Leftrightarrow \quad \Leftrightarrow \quad\left\langle x,(\delta)_{k=1}^{t}\right\rangle \succeq D .
\end{aligned}
$$

It is not difficult to check that the induced preference relation $\succeq^{\prime}$ satisfies the following properties:

1. No agreement is worse than disagreement: $(x, t) \succeq^{\prime} D$ for all $(x, t) \in X \times T$.

2. Time is valuable: $(x, t) \succeq^{\prime}(x, t+1)$ for every $(x, t) \in X \times T$.

3. Preferences are stationary: $(x, t) \succeq^{\prime}(y, t+1)$ if and only if $(x, 0) \succeq^{\prime}(y, 1)$ and $(x$, $t) \succeq^{\prime}(y, t)$ if and only if $(x, 0) \succeq^{\prime}(y, 0)$. 
4. Preferences are continuous: if $x_{n}, y_{n} \in X$ for all $n \in \mathbb{N}, x_{n} \rightarrow x \in X$ and $y_{n} \rightarrow y \in X$ and $\left(x_{n}, t\right) \succeq^{\prime}\left(y_{n}, t\right)$ for all $n$, then $(x, t) \succeq^{\prime}(y, n)$.

Property 1 follows from the analogous property of $\succeq$. Property 2 follows from monotonicity and compound independence of $\succeq$ : by monotonicity we have that $x \succeq\langle x$, $\delta\rangle$ and by repeated applications of compound independence we have that $\langle x$, $\left.(\delta)_{k=1}^{t}\right\rangle \succeq\left\langle x,(\delta)_{k=1}^{t+1}\right\rangle$, which means precisely that $(x, t) \succeq^{\prime}(x, t+1)$. Stationarity of preferences follows from repeated applications of compound independence and continuity of $\succeq^{\prime}$ follows from continuity of $\succeq$.

Therefore, the game $\Gamma(\delta)$ is equivalent to Rubinstein's (1982) game of alternating offers and time-preferences represented by $\succeq^{\prime}$ : they have the same set of players, and each player has the same strategy space and the same preferences over strategy profiles. Since the game of alternating offers and time preferences given by $\succeq^{\prime}$ has a unique subgame perfect equilibrium, which is the one described in the statement of the proposition (see Rubinstein, 1982), so does $\Gamma(\delta)$.

We can now state the following remark:

Remark 1. Within the alternating offers game $\Gamma(\delta)$, the behavior of a player who's preferences satisfy A1-A4 (in particular compound independence) is indistinguishable from the behavior of an expected utility maximizer.

Proof. Since preferences over $(X \times T) \cup\{D\}$ satisfy properties $1-4$ in the above proof, it follows from Fishburn and Rubinstein (1982) that there are functions $u_{i}: X \rightarrow \mathbb{R}_{+}$, for $i=1$, 2 , such that the auxiliary time-preferences can be represented by the utility function $U_{i}(x, t)=u_{i}(x) \delta^{t}$. But from Binmore et al. (1986) we know that the bargaining game with these time-preferences is equivalent to a model whose players have von Neumann-Morgenstern utility functions given by $u_{i}$, for $i=1,2$.

As a result of the above remark, all the known results that assume expected utility preferences extend to the case of preferences that satisfy compound independence. In particular, the limit of the subgame perfect equilibrium outcomes of $\Gamma(\delta)$ as $\delta$ goes to 1 is the ordinal Nash solution introduced in Rubinstein et al. (1992).

\section{Acknowledgements}

I thank the editor in charge for pointing out a mistake in a previous version. I also thank the referee for his useful comments.

\section{References}

Aumann, R.J., Brandenburger, A., 1995. Epistemic conditions for Nash equilibrium. Econometrica 63, 1161-1180. 
Binmore, K.G., Rubinstein, A., Wolinsky, A., 1986. The Nash bargaining solution in economic modeling. Rand Journal of Economics 17, 176-188.

Burgos, A., Grant, S., Kajii, A., 2002. Bargaining and boldness. Games and Economic Behavior 38, 28-51. Crawford, V., 1990. Equilibrium without independence. Journal of Economic Theory 50, 127-154.

Dekel, E., Safra, Z., Segal, U., 1991. Existence and dynamic consistency of nash equilibrium with nonexpected utility preferences. Journal of Economic Theory 55 (2), 229-246.

Fishburn, P.C., Rubinstein, A., 1982. Time preference. International Economic Review 23, 677-694.

Grant, S., Kajii, A., 1995. A cardinal characterization of the Rubinstein-Safra-Tomson axiomatic bargaining theory. Econometrica 63, 1241-1249.

Green, J., 1987. Making book against oneself, the independence axiom, and nonlinear utility theory. Quarterly Journal of Economics 102 (4), 785-796.

Hanany, E., Safra, Z., 2000. Existence and uniqueness of ordinal Nash outcomes. Journal of Economic Theory 90, 254-276.

LaValle, I.H., Wapman, K.R., 1986. Rolling back decision trees requires the independence axiom. Management Science 32, 382-384.

Nash, J.F., 1950. The bargaining problem. Econometrica 28, 155-162.

Osborne, M.J., Rubinstein, A., 1994. A Course in Game Theory. MIT Press, Cambridge.

Rubinstein, A., 1982. Perfect equilibrium in a bargaining model. Econometrica 50, 97-110.

Rubinstein, A., Safra, Z., Thomson, W., 1992. On the interpretation of the Nash bargaining solution and its extension to non-expected utility preferences. Econometrica 60, 1171-1186.

Safra, Z., Zilcha, I., 1993. Bargaining solutions without the expected utility hypothesis. Games and Economic Behavior 5, 288-306.

Sarin, R., Wakker, P., 1994. Folding back in decision tree analysis. Management Science 40, 625-628.

Segal, U., 1990. Two-stage lotteries without the reduction axiom. Econometrica 58 (2), 349-377.

Volij, O., 1996. Epistemic conditions for equilibrium in beliefs without independence. Journal of Economic Theory 70, 391-406.

Volij, O., Winter, E., 2002. On risk aversion and bargaining outcomes. Forthcoming in Games and Economic Behavior.

Wakker, P., 1988. Nonexpected utility as aversion to information. Journal of Behavioral Decision Making 1, $169-175$. 